\title{
Latest Trends in Telecommunication Standards
}

\author{
Xavier Costa-Pérez, Andreas Festag, Hans-Joerg Kolbe, Juergen Quittek, \\ Stefan Schmid, Martin Stiemerling, Joerg Swetina, Hans van der Veen \\ NEC Laboratories Europe, Heidelberg, Germany \\ \{surname\}@ neclab.eu
}

This article is an editorial note submitted to CCR. It has NOT been peer reviewed. The authors take full responsibility for this article's technical content. Comments can be posted through CCR Online.

\begin{abstract}
Standardization organizations play a major role in the telecommunications industry to guarantee interoperability between vendors and allow for a common ground where all players can voice their opinion regarding the direction the industry should follow. In this paper we review the current activities in some of the most relevant standardization bodies in the area of communication networks: 3GPP, IEEE 802.11, BBF, IETF, ONF, ETSI ISG NFV, oneM2M and ETSI TC ITS. Major innovations being developed in these bodies are summarized describing the most disruptive directions taken and expected to have a remarkable impact in future networks. Finally, some trends common among different bodies are identified covering different dimensions: i) core technology enhancements, ii) inter-organizations cooperation for convergence, iii) consideration of raising disruptive technical concepts, and iv) expanding into emerging use cases aiming at an increase of future market size.
\end{abstract}

\section{Categories and Subject Descriptors}

C.2 [Computer-Communication Networks]: Network Architecture and Design

\section{Keywords}

3GPP, 802.11, BBF, IETF, ONF, NFV, oneM2M and ITS

\section{INTRODUCTION}

The convergence of communications, computing and media content is bringing technologies from different industries together, pushing for the definition of common standards. Successful standards allow a large number of players to interoperate and innovate resulting in expanding commercial business, faster technology progress and end users enjoying wider choice range, richer functionality and lower costs.

In the telecommunications domain, given the complexity of the systems, no single company can cover the entire solution space. Naturally, in this domain standards for interoperability are a must. Moreover, the increasing system interdependencies are accelerating the collaboration between standards development organizations (SDOs) due to the fixed-mobile networks convergence trend. In parallel to this, the explosive increase in end user network utilization is rising the standards challenges bar, now required to ensure a satisfactory and coherent user experience across heterogeneous access networks.

In this paper we will review the trends in some of the most relevant standardization bodies in the area of communica- tion networks. Key innovations expected to have a major impact in future networks will be highlighted. Specifically, the following organizations will be covered:

- 3GPP The 3rd Generation Partnership Project for cellular networks.

- IEEE 802.11 for wireless local area networks.

- BBF The Broadband Forum for wireline networks.

- IETF The Internet Engineering Task Force for the Future Internet.

- ONF The Open Networking Foundation for SoftwareDefined Networking (SDN).

- ETSI ISG NFV The industrial specification group for Network Functions Virtualization (NFV).

- oneM2M for machine-to-machine communication.

- ETSI TC ITS for Intelligent Transportation Systems.

The set of bodies covered in this article is not to be understood as an exhaustive list, given the large number of standardization organizations in the world, but as a representative selection of the major ones in their respective areas according to the perspective of the authors of the article.

\section{3GPP}

The 3rd Generation Partnership Project (3GPP) [1] is neither an SDO nor a legal entity, but a collaborative project, in existence since 1998, between six regional SDOs (ARIB, ATIS, CCSA, ETSI, TTA, TTC), that has been developing reports and specifications for cellular technologies including 3.9G (LTE) and 4G (LTE-Advanced). Since LTE-A is a close evolution of LTE, the term LTE is commonly applied to LTE-A also and will be used as such in the rest of the paper.

The work in $3 \mathrm{GPP}$ is based on the concept of stages as defined by ITU Recommendation I.130. Another important tool is the "Release" concept, which allows for a stable platform for implementation and introduction of new features in the marketplace.

Release 11 was frozen in September 2012, with a number of exceptions still to be completed. For Release 12, Stage 1 was frozen in March 2013 with four exceptions. The plan for the remainder of Rel-12 is as follows:

- Stage 2 freezing target: December 2013

- Stage 3 freezing target: June 2014

Plans for Release 13 will start to be discussed during 2013. 


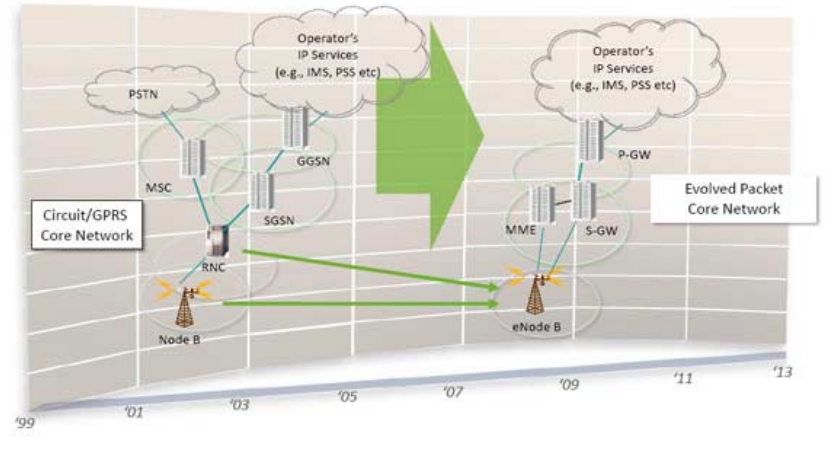

Figure 1: Illustration of 3GPP's Architecture Evolution. [Source: 3GPP, 2012]

\section{Selected Work Items}

3GPP continuously specifies enhancements and new solutions to help operators to cope with the evolving needs of its system and users. See Figure 1 for an illustration of 3GPP's architecture evolution. In the following a selection of work items is presented which adress some of these key challenges by defining new functionality planned to be incorporated in future releases:

The 3GPP RAN Sharing Enhancements (RSE) Study Item [3] of the System Architecture Working Group 1 (SA1) is currently defining new scenarios of multiple operators sharing network resources. The objective of the RSE Study Item is to create potential requirements that complement existing 3GPP system capabilities for sharing common RAN resources. The new scenarios aim to:

- Provide means to be able to verify that the shared network elements provide allocated RAN resources according to the sharing agreements and/or policies.

- Provide means for efficiently sharing common RAN resources according to identified RAN sharing scenarios (e.g. pooling of unallocated radio resources).

- Provide means to flexibly, dynamically and automatically allocate RAN resources on-demand at smaller timescales than the ones supported today.

The paradigm change represented by the 3GPP acceptance of automated means to allocate RAN resources dynamically and on-demand deserves special mention. This enhanced functionality opens the door to new industry players as well as to an evolution of operators' business models which could potentiall have a deep impact in the industry [4].

Proximity based services (ProSe): This study collects use cases and creates a set of potential requirements for operator network controlled discovery and communications between UEs that are in proximity, under continuous network control, and are under 3GPP network coverage, for:

- Commercial/social use

- Network offloading

- Public Safety

- Integration of current infrastructure services, to assure the consistency of the user experience including reachability and mobility aspects
The communication path can be established directly between the UEs or routed via local eNB(s). UEs may also use WLAN for communication. Additionally, the study item will study use cases and identify potential requirements for Public Safety, in case of absence of EUTRAN coverage.

Group communications over LTE (GCSE_LTE) is a key functionality of land mobile radio (LMR)/professional mobile radio (PMR) and public safety systems. Sources of input requirements are: NPSTC - an organization made up of all the major public safety organizations in the US, The Tetra + Critical Communications Association (TCCA) which is considering LTE for critical communications and the International Union of Railways (UIC) which is considering LTE as the basis of the future generation after GSM-R.

Regarding the integration of Single Sign-On (SSO) frameworks within 3GPP networks, a work item (SSO_int) has been started which covers the following:

- Service requirements for integration of Identity Management and SSO frameworks, e.g. OpenID

- Service requirements for Operators to enable users to access 3rd party services using Operator controlled user credentials

- Service requirements associated with ensuring that the intended user is making use of the associated SSO capability (including the case when the UE has been stolen or lost)

User Plane Congestion Management: In order to assist operators during traffic peak times, which cause user plane congestion in the radio access and/or backhaul network, 3GPP has started to evaluate novel mechanisms to handle the traffic in a way that improves the overall quality of experience for mobile users and/or allow the system to admit more active users with an adequate level of service quality. An important requirement for the management of user plane congestion is that operators should be able to flexibly control the mitigation measures through policies in order to enable service differentiation.

Traffic offloading to WLAN networks: Although basic support to connect via WLAN based access networks has been standardized many years ago, current standards work strives to provide operators efficient control to offload data traffic to WLANs. A central element in this framework is the Access Network Discovery and Selection Function (ANDSF), a new control entity that allows operators to provide (push or pull) access network discovery, selection and offloading policies to the mobile terminals. Rel-12 work focuses on the coordination of policies provided by the WLAN access networks directly (e.g. via HotSpot 2.0) and the ANDSF, and improves the granularity of the traffic offload by supporting policies on the basis of IP flows. 3GPP is also enhancing WLAN capable mobile terminals and the network to support new traffic offload modes:

- Non-Seamless WLAN Offload triggers mobile terminals to utilize the WLAN interface for data traffic, once a WLAN access network is available. This allows traffic offload to the the local WLAN network without any mobility support.

- Offload via Trusted WLAN Access Network allows operators to authenticate the subscribers and to tunnel the offloaded traffic back to the operator core network 
in order to offer subscribers also access to operatorprovided services (e.g. IMS) via the offload network, and also to support Policy Control and Charging. The focus of Rel-12 standards work in this area is on providing seamless mobility support and simultaneous access to multiple Packet Data Networks.

Traffic offload to femtocells or small cells networks: Support for traffic offload from macro cells to femtocells (i.e. HNBs/HeNBs), in order to reduce macro-cellular network load, has already been standardized in Rel-10. Ongoing standards work further enable operators to offload Internet traffic directly at the femtocells or at a local gateway in a small cell network in order to reduce the traffic and processing load on backhaul and core network links as well as on network entities.

With respect to the Radio Access Network, a 3GPP workshop was held in June 2012 [5] where the following requirements were identified for Release 12 and beyond:

- Capacity increase to cope with traffic explosion

- Energy saving

- Cost efficiency

- Support for diverse application and traffic types

- Higher user experience/data rate

- Backhaul enhancement

In particular, a need for enhanced support for small cells for LTE was emphasized. To achieve this, the following technologies would be reviewed:

- Interference coordination / management

- Dynamic TDD

- Enhanced discovery / mobility

- Frequency separation between macro and small cells with higher frequency band, e.g. $3.5 \mathrm{GHz}$ band, for the small cells

- Inter site Carrier Aggregation / macro cell assisted small cells

- Wireless backhaul for small cell

Many players also expressed a clear interest in improving LTE multi-antenna/site technologies, such as 3D MIMO, beamforming, CoMP (Coordinated Multi-Point) and support for diverse data traffic types, such as control signalling reduction.

After the workshop, the scenarios and requirements for small cell enhancements were reviewed and approved in Technical Report TR 36.932 [6]. Subsequently two studies on small cell enhancements were started, covering physical layer aspects and higher layer aspects respectively. In parallel, studies have been started on several other areas identified at the workshop, such as energy saving, device-to-device proximity services, and a 3D-channel model for elevation beamforming and FD-MIMO studies. In addition normative work has also been started, on downlink-uplink interference management and traffic adaptation, heterogeneous network mobility enhancements and inter-band and intra-band carrier aggregation for a number of frequency bands.

\section{IEEE 802.11}

The IEEE 802.11 standards [7] have developed a mass market, with an impressive penetration share in homes, offices, hotspots and public areas. Nowadays, many devices incorporate wireless LAN technology, e.g., laptops, tablets, mobile phones and media players. With low-cost chipsets and support for high data rates, 802.11 has become a universal solution for an ever increasing application space. As a direct consequence of its high market penetration, several amendments to the basic 802.11 standard have been developed in the past years, see e.g., [8,9], and more are under development. Their purpose is to fix technology issues and/or add missing functionality according to forecasted market demands. See Figure 2 for an illustration of the 802.11 architecture. In the following we provide an overview of the emerging 802.11 future standards driven by several active Task Groups (TGs)[7]:

- TGm: This group provides maintenance of the IEEE 802.11 standard by rolling published amendments into revisions of the 802.11 standard.

- TGac: Specifies enhancements to the $802.11 \mathrm{MAC}$ and PHY to support very high throughput (500-1000 Mbps) in the $5 \mathrm{GHz}$ bands (Expected completion date Nov. 2013). This specification is the natural substitute of $802.11 \mathrm{n}$ and expected to become ubiquitous in the future.

- TGad and TGaj: TGad defined standardized modifications to both the 802.11 physical layers (PHY) and the 802.11 Medium Access Control Layer (MAC) to enable operation in the $60 \mathrm{GHz}$ frequency band (typically $57-66 \mathrm{GHz}$ ) capable of very high throughput $(\geq$ $1 \mathrm{~Gb} / \mathrm{s})$. It also enables fast session transfer between 802.11 PHYs. The specification has been completed and is expected to be published in early 2013. TGaj is the chinese market specific effort for supporting very high throughput in the China millimetre-wave bands including the 59-64 GHz and $45 \mathrm{GHz}$ frequency bands to enable multi-Gbps throughput at low power.

- TGaf: An amendment that defines modifications to both the 802.11 physical layers (PHY) and the 802.11 Medium Access Control Layer (MAC) to meet the legal requirements for channel access and coexistence in the TV White Space.

- TGah: An amendment that defines operation in Sub $1 \mathrm{GHz}$ frequencies. Supports applications that benefit from range extension, such as smart grids and M2M services.

- TGai: An amendment that supports Fast Link Setup, i.e., to reduce time to set up an association.

- TGaq: Defines mechanisms that assist in pre-association discovery (PAD) of services by addressing the means to advertise their existence and enable delivery of information that describes them.

Based on these activities two major trends can be identified within the IEEE 802.11 WG. First, most TGs work on improving the mainstream standard performance.TGac, TGad and TGaj are aimed at fulfilling the International Telecommunication Union's (ITU's) requirements on proposals for the IMT Advanced standard [10]. They target 


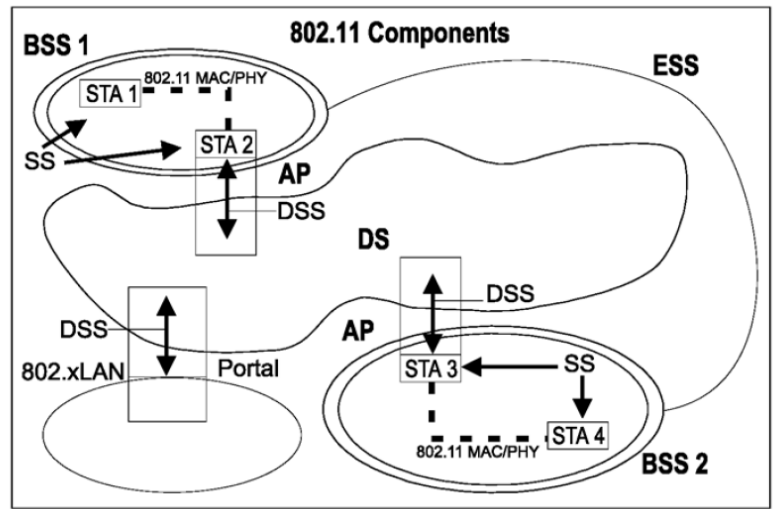

Figure 2: Illustration of the IEEE 802.11 architecture. [Source: IEEE 802.11-2012 Std.]

greater than $1 \mathrm{~Gb} / \mathrm{s}$ throughput, but while 802.11ac considers the traditional WLAN frequencies below $6 \mathrm{GHz}, 802.11 \mathrm{ad}$ and 802.11aj apply to the millimetre-wave bands. In the TGai and TGaq cases, the aim is to improve the 802.11 link setup process to connect to APs and reduce the discovery process overhead such that emerging use cases with an increasing number of access points in range and/or higher user mobility can be efficiently addressed (e.g., metro stations, shopping malls).

The second major trend focuses on exploiting additional frequency bands expected to be made available for 802.11 . Two options are considered in this case: white spaces (TGaf) and sub $1 \mathrm{GHz}$ bands (TGah). The aim of these approaches is, on the one hand, expand the range available for these kind of networks and, on the other hand, entering into new potential markets (e.g., smart meters).

Finally, future 802.11 task groups are usually proposed at the WNG group. At the moment of writing this article one Study Group has been formed which may eventually form a new Task Group: General Link (GLK) which aims at defining an 802.11 solution to enhance the ability of IEEE 802.11 media to provide internal connections as transit links within IEEE Std 802.1Q bridged networks.

\section{BROADBAND FORUM}

The Broadband Forum (BBF) [11] is an industry consortium that was established back in 1994 as the ADSL Forum. It was later re-named to DSL Forum and finally in 2009, during the merger with the IP/MPLS Forum, became the BBF. As of today, it consists of approximately 180 service providers, vendors, consultants, academic institutes and test labs. The BBF sees its main tasks in engineering network technology solutions to provide economical and effective broadband deployments, leveraging its CWPM (CPE WAN Management Protocol) suite of remote-management protocols/specifications, and using where possible other existing standards by profiling them. See Figure 3 for an illustration of the BBF scope.

Having started with technical reports focusing on broadband access line technologies, the forum made a significant step in widening its scope towards network architectures with the release of the TR-59 and later TR-101 architecture documents, providing a blueprint for building fixed line broadband access networks ranging from customer premises

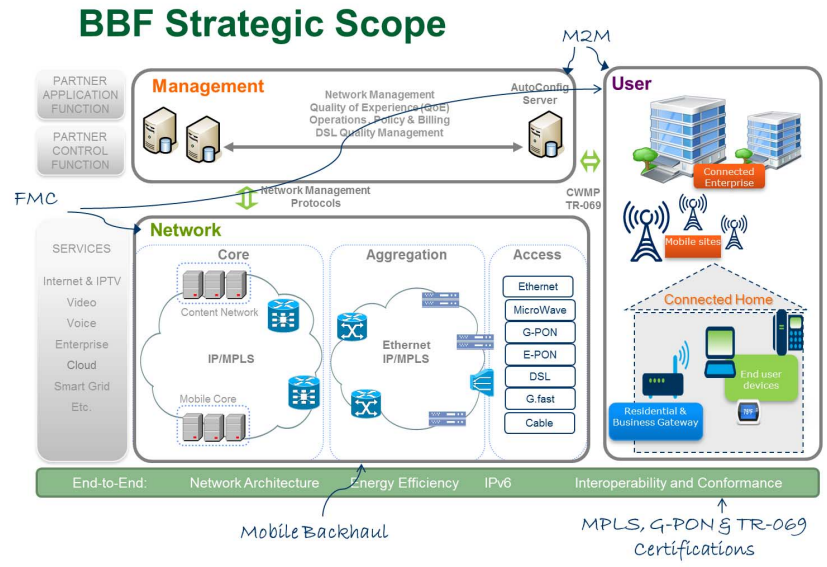

Figure 3: Illustration of the Broadband Forum Scope. [Source: BBF, 2012]

towards the IP edge. Having merged with the IP/MPLS Forum, the BBF is now able to provide an end-to-end scope in broadband networks. In the case of home network management, the BBF made a significant step beyond profiling standards, by defining its own TR-069 protocol suite and data models for home network management. Still, the BBF does not plan to define protocols unless a real gap exists.

The forum as of today is structured in multiple technical working groups dealing with home networks, end-to-end architectures, the IP/MPLS and core, metallic transmission technologies, fibre access and network management. Besides that, a marketing group disseminates BBF's work into the industry and a service provider action council (SPAC) is in place to drive the directions of the BBF's work in order to fulfill the needs of the network operators. Recently, a new group SIMR (Service Innovation \& Market Requirements) was created to foster long-term planning of new projects.

In the Broadband Home working group, the BBF mainly addresses the management of home networks to provide services beyond simple network connectivity through the residential gateway (RG). The developed data models are purposebuilt for TR-69 CWMP and are based on requirements collected in TR-124v3 (Functional Requirements for Broadband Residential Gateway Devices) for in-home networks. Assured service delivery for multimedia services such as IPTV is currently one of the key work items, with a focus towards measuring performance inside the progressively more complex home networks. Going beyond classical service provider business, the BBF has started significant work, and is becoming one of the key players, in the machine-to-machine communications (M2M) space. It is currently studying requirements for device management proxies, defining appropriate data models to e.g. access sensors and actuators using various $\mathrm{LAN}$ technologies (Zwave, ZigBee, etc). A major goal is to identify/define means to abstract a set of higherlevel control and signaling commands which are independent of the devices' access technology.

The End-to-End (E2E) working group addresses network architectures with a strong focus on dealing with subscribers and session handling, including the transition from IPv4 to IPv6. The BBF has just finished work on a new network architecture (TR-145) and has moved on to cover the requirements inside the key network elements in this ecosys- 
tem. In the new architecture, the BBF describes a more distributed subscriber edge node architecture, de-composing the former BRAS (Broadband Remote Access Server) into a BNG (Broadband Network Gateway) and a BSG (Broadband Service Gateway). This allows bringing Layer-3 and MPLS functionality into the access networks.

An important milestone for the BBF was the establishment of a strong collaboration with the $3 \mathrm{GPP}$ in the area of fixed mobile convergence. After finally agreeing on basic principles of policy control inside the BBF network (TR-134), the BBF was able to define interworking principles to interconnect fixed and mobile networks (TR-203). This provides coherent QoS and charging features for $3 \mathrm{GPP}$ handsets when roaming into fixed line access networks, e.g. as in the femto cell and WLAN access cases. Current work items impact interworking and converged scenarios where a 3GPP PCRF controls also the BBF network. In the IP/MPLS and Core group, the main work item is on MPLS for the mobile backhaul (TR-221). This document is being further developed, now also taking into account evolving backhaul architectures and transport for supporting small cells deployments and considerations for applying energy efficiency technologies. Additionally, IP/MPLS and Core has published TR-223 on MPLS over Aggregated Interfaces (MPLSoAI). Currently the working group is finalizing a set of test suites for MPLS over ATM and MPLS and for Ethernet services over MPLS, while at the same time is working towards further test suites for MPLS OAM. IP/MPLS and Core working group are also working closely with MEF on developing a technical specification for MPLS in Carrier Ethernet Networks.

Both the Fibre Access Network group as well as the Metallic Transmission group focus on testing procedures and plans, certification and energy efficiency driven, e.g., by the European code of conduct on Energy Consumption of Broadband Equipment. Both groups interact very closely with other SDO such as FSAN and the ITU. For GPON, the $\mathrm{BBF}$ has recently launched a certification programme. In the metallic access space, DSL vectoring is one of the key topics. Bringing both access types, fibre and copper together, the BBF has started a big project on FTTdP (Fibre to the distribution point). In FTTdP, fibre terminates directly in front of the houses while connectivity over the last few meters is provided using existing copper lines. This new technology impacts many BBF groups, including E2E.

Finally, the Operations and Network Management group deals with managing nodes and CPE equipment in access networks. The scope of this group includes performance testing as well as providing a bridge to OSS/BSS focused SDO such as the TM Forum. To react more quickly, or even proactively, according to new trends in the industry, the newly set up SIMR group deals with new service requirements and will provide business level requirements and guidance to the focus of the other groups' technical work. The SIMR group is currently investigating requirements for supporting cloud computing on the BBF architecture and is studying SDN-based use cases for broadband networks.

\section{INTERNET ENGINEERING TASK FORCE}

The Internet Engineering Task Force (IETF) [12] is an organization that develops standards for the Internet through the participation of individual contributors in an open process. The standards and also further informational docu- ments are freely accessible on the Internet and so is also the whole standards process. The probably most known and used protocols out of the IETF are, for instance, HTTP/1.1 [13] and the Multiprotocol Label Switching (MPLS) [14]. IETF protocols are widely used in the public Internet and also by other standards organizations. Readers interested in the overall structure are referred to the IETF web page [12] for detailed information. In the following some IETF selected trends are introduced.

Probably the most used and known IETF protocol is the Hypertext Transfer Protocol (HTTP) [13] which is used to carry a variety of different applications on top of it, for instance, HTML-based web pages. The current HTTP/1.1 specification is now 15 years old and the usage of HTTP has dramatically changed from the delivery of pure text and picture web pages, over dynamically web pages to web-based applications, such as, video conferencing in the browser (see also later about RTCWEB in that respect). This is the starting point of the HTTPbis working group [15] that works on an update of the existing HTTP/1.1 specification to address ambiguities as well as on a potential successor that retains the semantics of $\mathrm{HTTP} / 1.1$ but with a different syntax to overcome performance limitations.

IP-based voice and video real-time services are typically run as hardware devices, e.g., the classical desk phone, or as software application, e.g., a Voice over IP (VoIP) client on your computer. However, there are several implementations of these services in web browsers that implement voice call or video chat services without requiring a separate software application to be installed. This type of real-time application is commonly called Real-Time Communication in WEB-browsers (RTCWEB). The World Wide Web Consortium (W3C) and the RTCWEB working group in the IETF [16] are jointly developing standards to enable interoperable browser-to-browser voice and video calls. The W3C is taking care of the browser-side required to enable such a service, while the IETF is in charge of the real-time and transport protocols being used between the browsers.

The topic of Software Defined Networking (SDN) is discussed in the IETF for a long time and eventually led to the creation of a Software Defined Networking Research Group (SDNRG) activity in the Internet Research Task Force. The SDNRG explores the space of Software Defined Networking in terms of programability of networks on all layers, e.g., forwarding and control planes, changes in the transport networks, service layers, and the orchestration of all of these. In parallel, the IETF discusses the creation of an Interface to the Internet Routing System (I2RS) working group [17]. The proposed I2RS protocol should allow applications to access, install, delete, modify, or learn about the routing state on classical IP routers. I2RS would be another candidate for a so-called south-bound interface between a network controller and a network element. Another candidate for such a south-bound interface is OpenFlow 6, where it should be noted that Openflow access the flow tables of switches, but not the routing tables.

\section{OPEN NETWORKING FOUNDATION}

The Open Networking Foundation (ONF) [18] is an industry forum that develops standards for an architecture called Software-Defined Networking (SDN). The basic principle of $\mathrm{SDN}$ is the separation of control plane and data plane in routers, switches, and similar devices. Conventionally, the 
fast packet forwarding (data path) and the high level routing decisions (control path) occur on the same device. OpenFlow separates these two functions. The data path portion still resides on an OpenFlow switch, while high-level routing decisions are moved to a separate OpenFlow controller, typically running on a standard server. The OpenFlow switch and controller communicate via the OpenFlow protocol, which defines messages, such as packet-received, send-packet-out, modify-forwarding-table, and get-statistics.

Potential impact of the OpenFlow can be two-fold. It may change the common packaging of today's routers where packet forwarding functions are bundled with routing functions in the same device. Even more importantly, it is targeted at changing the way networks are deployed and operated. Having the control plane implemented in software on standard servers enables a much higher innovation rate for networks. Control functions can be deployed, updated, and replaced by just managing software in a cloud computing environment without touching or replacing hardware boxes that are located where packet forwarding functionality is needed.

The ONF develops standards needed for realizing this SDN vision. It standardizes the OpenFlow protocol and a protocol called OF-CONFIG for managing OpenFlow capable switches. It creates documents dealing with designing, testing, and operating SDN devices and networks. Beyond such guidelines, the ONF produces test specifications for OpenFlow-capable equipment and specifies an OpenFlow certification process, that will soon be available. The first version 1.0 of the OpenFlow protocol was developed at the Stanford University. Since version 1.1 the ONF is the home of OpenFlow. After that, the ONF has published versions 1.1, 1.2, 1.3, and 1.3.1. With each protocol version the OpenFlow functionality gets extended. The main improvement of version 1.1 was support for MPLS labels. Version 1.2 added support for IPv6 and introduced a more flexible packet matching and version 1.3 added support for traffic shaping.

Current work focuses on the modularization and generalization of the OpenFlow protocol. Modularization will specify subsets of OpenFlow functions that can be included or excluded from different implementations. It also provides a cleaner way for adding proprietary extensions. The generalization will allow for better modeling of particular switch hardware in the OpenFlow protocol. Version 1.0 is the most commonly implemented version, oftenly implemented with proprietary extensions covering features that have been added to the standard in versions 1.1 and 1.2. Now, Version 1.3.1 is expected to become again adopted by many implementers.

The configuration protocol OF-CONFIG uses the IETF's NETCONF protocol for configuring OpenFlow switches, particularly before they connect to a controller via the OpenFlow protocol. OF-CONFIG version 1.0 provided the basic functions, version 1.1 added further capabilities, such as, for example, configuration of tunnel endpoints. Next steps in this area will be providing operations, administration and management (OAM) functions for OpenFlow networks, such as event notifications, link monitoring, path monitoring, and topology detection.

\section{ETSI ISG NFV}

In autumn 2012 a group of telecom operators from Asia,
Europe, and the US announced a new Industrial Specification Group (ISG) at the European Telecommunications Standards Institute (ETSI). The focus of this new ISG is on Network Functions Virtualization (NFV) [19].

The basic idea of NFV is implementing data plane network functions on top of a virtualization platform consisting of servers, switches, and storage, which could be located in data centers, network nodes and end user premises. It is assumed that all data plane network functions, such as e.g. a firewall, a carrier-grade NAT, an SGSN/GGSN, a BRAS, DPI and CDN functions can be virtualized on such a platform.

Operators expect the following benefits from networks having all data plane functions virtualized:

- Lower cost of equipment

- Improved scalability of network functions

- Reduced cost and power consumption through function consolidation and sharing of hardware resources across different functions, services, and customer groups

- Fully remote (zero-touch) deployment of appliances

- Spatial function migration with no or very short service interruption

- Shorter innovation cycles

- More open market for virtual appliances than today for hardware-bound appliances

For achieving these goals, several technical challenges must be overcome. The obvious first challenge is virtualizing implementations of network functions and providing them with remote deployment and configuration capabilities. Then, scale-out and scale in support needs to be added. This can be difficult for some functions, e.g., firewalls, that need splitting of traffic and load balancing when it is scaled up from a single instance to multiple instances of virtual machines that jointly implement a single network function. If virtualized functions get relocated from their original location in the network into a data center, the traffic handled by this function needs to be re-directed to the instance of the function in the data center. For some virtualized functions it is important to trace its location. OAM functions will need to be extended for providing this new required functionality.

Solving the issues listed above adds a lot of complexity. For handling it, an orchestration of virtualized functions itself and of traffic re-direction is needed.

Work in the new ETSI ISG is not intended to produce many normative standards, but rather to analyze issues arising from the introduction of NFV and to give recommendations and guidelines for designing networks with virtualized functions. In case the ISG identifies a concrete need for new standards, e.g., a new protocol, the ISG intends to request an already established standards body to produce the needed standard.

The ISG has been founded by operators only, but is open to other organizations. The kick-off meeting in January 2013 was attended by participants from 52 different companies. Initially, the ISG created four working groups (WGs) and two expert groups (EGs) covering cross topics:

- Architecture of Virtualization Infrastructure WG

- Management \& Orchestration WG

- Software Architecture WG 


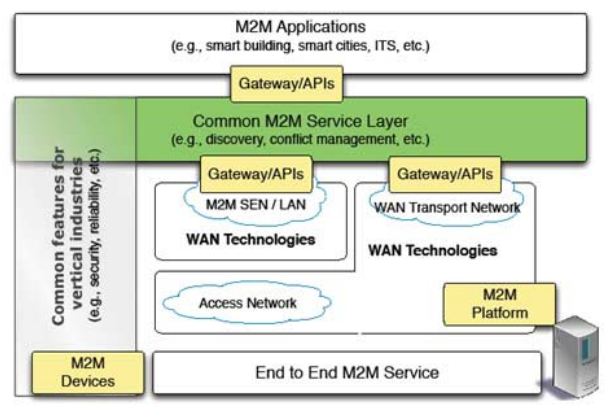

Figure 4: Positioning of the oneM2M standard

- Reliability \& Availability WG

- Performance \& Portability EG

- Security EG

The ISG plans for a rather short lifetime of 18 months and for completion of all work items already in 2014 .

\section{ONEM2M}

Today many industries are addressing highly specialized Machine-to-Machine (M2M) solutions that often need customized hardware and software. Such specialized solutions typically cause high costs, CAPEX as well as OPEX. Thus there is a strong need to develop a horizontal common M2M service layer standard that is supporting a large number of industry verticals and which can foster the development of a global M2M market.

The oneM2M Global Initiative [20] is a partnership project, established mid 2012, in order to co-operate in the production of globally applicable, access-independent M2M Service Layer specifications related to M2M Solutions. The common service layer is intended to be embedded within various hardware and software components to ensure M2M devices can communicate on a global scale. OneM2M had been initiated by the seven global telecom SDOs: ARIB, TTC (Japan); ATIS, TIA (USA); CCSA (China); ETSI (Europe); and TTA (Korea). Currently, about 220 participating partners and members are registered. See Figure 4 for an illustration of the oneM2M positioning.

The main objective of oneM2M is to develop one globally agreed set of M2M specifications. For this, oneM2M plans to consolidate current existing M2M service layer standards activities. OneM2M collaborates with various SDOs and industry fora in charge of developing vertical market aspects of M2M applications. More specifically, oneM2M targets on the following benefits to the M2M ecosystem:

- Boost M2M economies of scale and shorten time-tomarket

- Simplify development of applications

- Leverage a worldwide network for enhanced potential of services and to expand business opportunities

- Reduce standardization overlap and confusion and provide ongoing standards support

\section{COOPERATIVE ITS}

Current directions in Intelligent Transport Systems (ITS) target at making transport vehicles, in particular cars in road traffic, cooperate by communication, in contrast to 'sensing' their environment (e.g. by radar) as in today's vehicles. WLAN is particularly considered as a communication technology for vehicular environments since it enables a direct and frequent data exchange among vehicles and between vehicles and roadside infrastructure. To improve road safety and traffic efficiency a dedicated spectrum in the $5 \mathrm{GHz}$ band has been allocated in the US and Europe for these applications.

An IEEE 802.11 variant for vehicular communication formerly known as amendment IEEE 802.11p, referred to 'Wireless Access in Vehicular Environments' (WAVE) in the US and 'ITS G5' in Europe - has been developed and adopted for the US and European road safety systems. WAVE and ITS-G5 cover the PHY and MAC layers and operate in a dedicated spectrum at the $5.9 \mathrm{GHz}$ frequency band allocated for road safety-related applications: $75 \mathrm{MHz}$ in 7 channel in the US; $30 \mathrm{MHz}$ in 3 channels for ITS-G5A and additional channels (G5B and G5C) in Europe. WAVE/ITS G5 is very similar to the amendments known from IEEE 802.11a. In order to cope with the increase inter-symbol interference in vehicular environments, they have halved the OFDM timing parameters which result in the $10 \mathrm{MHz}$ channels and half data rate compared to IEEE 802.11a. Further extensions include the communication outside the context of a BSS (OCB), which avoids time-consuming signaling procedures for direct and rapid data exchange.

In the US, IEEE has developed the 1609 series of standards, also referred to as Dedicated Short Range Communication (DSRC) [21]. Core of the standard family is the WAVE Short Message Protocol (WSMP) (Part 3 of IEEE1609). WSMP is a network protocol that supports single-hop communication and is optimized for a minimum size of its packet header. Further parts of the standards define management activities in part 1 , the security protocols in part 2, and extensions to the physical channel access for multi-channel operation defined in part 4 . On top of the WSMP, application messages are defined by the SAE 2735 standard. The Basic Safety Message (BSM) is a periodic message that is carried in the payload of the WSM. Other messages are for infrastructure communication (MAP for intersection topology, SPAT for signal phase and timing) or probe vehicle data.

In Europe, ETSI TC ITS [22] and CEN TC 278 develop a series of standards for cooperative ITS [23]. See Figure 5 for an illustration of the ETSI TC ITS scope. Relying on ITS G5, an ad hoc network layer protocol for singlehop and multi-hop communication, GeoNetworking (EN 302 636), utilizes geographical positions for communication: (i) addressing of geographical areas in which a packet should be distributed, more precisely to address nodes in an area without using their node identifier and (ii) in the forwarding process in order to make the packet routing efficient and scalable. Application message protocols (ETSI EN 302 637) are the Cooperative Awareness Message (CAM) for periodic, single hop messages and the Distributed Environmental Notification Message (DENM) for event-driven, multi-hop messages.

More standards are being developed by other standardization development organizations (SDOs), such as ISO TC 


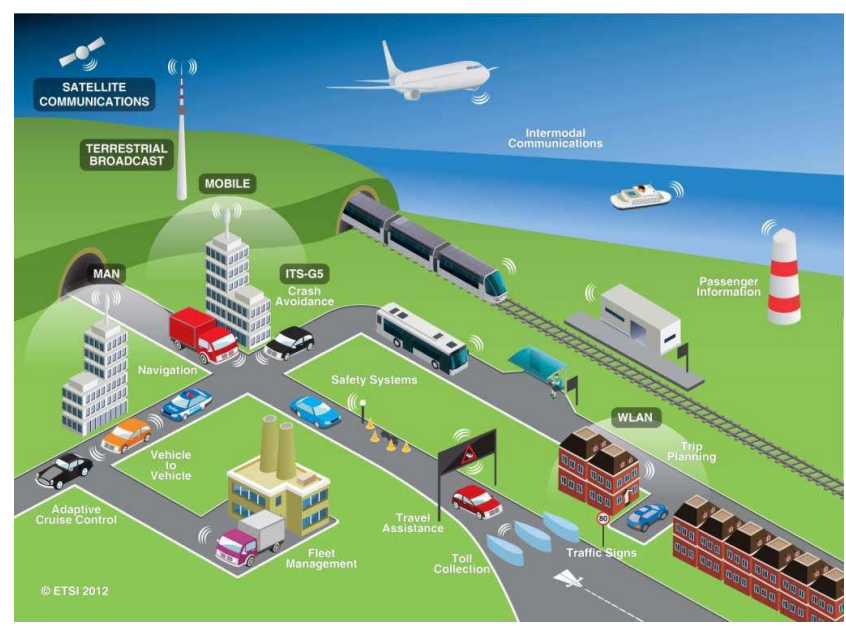

Figure 5: Illustration of ETSI TC ITS application area scope. [Source: ETSI, 2012]

204, ITU ARIB, etc. In order to avoid duplicate regional standards by different SDOs, efforts for harmonization of standards, in particular between EU and US, are undertaken. The standards are tested in major field trials to assess the impact of cooperative ITS on safety and traffic efficiency, such as the DRIVE C2X project in Europe and the Safety Pilot in the US. Market introduction of the system is expected in the next years by a regulatory decision of the US DoT in 2013 for the US and based on a Memorandum of Understanding (MoU) signed by the vehicle manufacturers in the Car-2-Car Communication Consortium, a European industry consortium, after 2015.

\section{SUMMARY AND CONCLUSIONS}

In this paper we reviewed the trends in some of the most relevant standardization bodies in the area of telecommunication networks: 3GPP for cellular networks, IEEE 802.11 for wireless local area networks, BBF for wireline networks, IETF for the Future Internet, ONF for software-defined networks, ETSI ISG NFV for network functions virtualization, oneM2M for machine-to-machine communications and ETSI TC ITS for intelligent transportation systems. Major innovations being developed in these bodies have been summarized describing the most disruptive directions taken and expected to have a remarkable impact in future networks roll-out.

In general, some key common trends have been identified, comprising several bodies within related networking areas: i) given the forecasted mobile Internet traffic explosion, wireless technologies are spending most efforts in dramatically increasing future peak data rates, ii) fixed and mobile networks market convergence is closely followed by the standardization organizations to guarantee a successful interworking by establishing strong collaboration ties, iii) new disruptive technological concepts as Software-Defined Networking are seriously considered by the organizations for future specifications and iv) emerging use cases such as M2M and ITS, expected to increase the telecommunications market size, are being accomodated inside and/or outside existing organizations as required by defining their own specifications.

\section{REFERENCES}

[1] Third Generation Partnership Project (3GPP) at http://www.3gpp.org/

[2] 3GPP Technical Specification Group Services and System Aspects; Network Sharing; Architecture and Functional Description. 3GPP TS 23.251, Sep. 2011.

[3] 3GPP System Architecture Working Group 1 (SA1): RAN Sharing Enhancements Study Item at http://www.3gpp.org/ftp/Specs/html-info/WiSpec540028.htm

[4] 3GPP System Architecture Working Group 1 (SA1), Use case for On-demand Automated Capacity Brokering, S1-124050, November, 2012.

[5] 3GPP TSG-RAN Workshop on Release 12 and onward, Future Radio on 3GPP, June 2012.

[6] 3GPP Technical Specification Group Radio Access Network; Scenarios and Requirements for Small Cell Enhancements for E-UTRA. 3GPP TR 36.932, Dec. 2012.

[7] IEEE 802 Working Group 11 at http://www.ieee802.org/11/

[8] G. Hiertz et al. The 802.11 Universe. IEEE Communications Magazine, January 2010.

[9] X. Pérez-Costa and D. Camps Mur. IEEE 802.11e QoS and Power Saving Features: Overview and Analysis of Combined Performance. IEEE Wireless Communications Magazine, August 2010.

[10] International Telecommunications Union (ITU), Requirements Related to Technical Performance for IMT-Advanced Radio Interface(s), Report M.2134, November 2008.

[11] The Broadband Forum (BBF) at http://www.broadband-forum.org/

[12] IETF main page at https://www.ietf.org/

[13] R. Fielding et al., Hypertext Transfer Protocol HTTP/1.1, RFC 2616, https://datatracker.ietf.org/doc/rfc2616/, June 1999.

[14] E. Rosen, A. Viswanathan, and R. Callon, Multiprotocol Label Switching Architecture, RFC 3031, https://datatracker.ietf.org/doc/rfc3031/, Jan. 2001.

[15] HTTPbis Working Group Charter at https://datatracker.ietf.org/wg/httpbis/charter/

[16] RTCWEB Working Group Charter at https://datatracker.ietf.org/wg/rtcweb/charter/

[17] I2RS mailing list, https://www.ietf.org/mailman/listinfo/i2rs

[18] The Open Network Foundation web page at http://www.opennetworkingfoundation.org/

[19] ETSI ISG on Network Functions Virtualization (NFV), http://portal.etsi.org/portal/server.pt/community/NFV/367

[20] The oneM2M Global Initiative at http://www.onem2m.org/

[21] J. B. Kenney: Dedicated Short range Communications (DSRC) Standards in the United States, Proceedings of the IEEE , Vol. 99, No. 7, July 2011

[22] ETSI ITS website: http://www.etsi.org/technologiesclusters/technologies/intelligent-transport

[23] S. Hess etal.: Towards Standards for Sustainable ITS in Europe, 16th ITS World Congress and Exhibition, Stockholm, Sweden, September 2009. 